\title{
Quand monnaies et médailles s'offrent un nouveau parcours
}

\section{Dominique Antérion}

\section{(2) OpenEdition}

\section{Journals}

Édition électronique

URL : http://journals.openedition.org/artefact/2316

DOI : $10.4000 /$ artefact.2316

ISSN : 2606-9245

Éditeur :

Association Artefact. Techniques histoire et sciences humaines, Presses universitaires du Midi

Édition imprimée

Date de publication : 6 décembre 2018

Pagination : 303-313

ISBN : 978-2-8107-0595-5

ISSN : 2273-0753

\section{Référence électronique}

Dominique Antérion, «Quand monnaies et médailles s'offrent un nouveau parcours », Artefact [En ligne], 8 | 2018, mis en ligne le 21 juin 2019, consulté le 27 novembre 2020. URL : http:// journals.openedition.org/artefact/2316 ; DOI : https://doi.org/10.4000/artefact.2316

\section{(c) (i) $\odot$}

Artefact, Techniques, histoire et sciences humaines est mise à disposition selon les termes de la Licence Creative Commons Attribution - Pas d'Utilisation Commerciale - Pas de Modification 4.0 International. 


\section{Quand monnaies et médailles s'offrent un nouveau parcours}

\section{Dominique Antérion}

\section{Résumé}

Le musée du 11 Conti-Monnaie de Paris inauguré le 25 septembre 2017, est le troisième depuis 1833 à avoir été aménagé sur le site de l'Hôtel de la Monnaie (Paris $6^{\mathrm{e}}$ ). Totalement différent des deux précédents, il propose au public un cheminement thématique où s'enchaînent successivement l'histoire et la physique des matières premières, l'histoire des différentes méthodes de gravure et de frappe, la présentation de l'Établissement monétaire de Pessac, les fonctions et usages de la monnaie, l'art de la collection, les trésors et les œuvres d'exception dans les domaines de la monnaie, de la médaille ou encore du bijou. Riche d'un parc multimédia important, ce musée témoigne aussi et surtout de la richesse et de la diversité des collections de la Monnaie de Paris dont l'importance - due à plusieurs siècles d'enrichissement - et la gestion au quotidien vont bien au-delà du seul parcours permanent.

\section{Mots-clés}

musée, muséographie, Monnaie de Paris, collections, monnaie, médaille, multimédia

9 Dominique Antérion, « Quand monnaies et médailles s’offrent un nouveau parcours », Artefact, 8, 2018, p. 303-313. 


\section{When coins and medals are offered a new exhibition path}

\section{Abstract}

The 11 Conti-Paris Mint Museum inaugurated on September $25^{\text {th }}$ of 2017 , is the third one to inhabit the Hôtel de la Monnaie building in Paris' $6^{\text {th }}$ arrondissement. Completely different from the two former museums, it takes the public through a thematic path successively leading to raw material History and physics, and to the History of engraving and coinage methods. It also shows Pessac's Monetary Establishment, money's functions and uses, the art of collecting, treasures, and extraordinary artworks within coins, medals or jewelry collections. With significant multimedia contents, the museum is also a great and rich heritage as shown by the collections of the Mint's museum. The importance of these collections, which have been incremented for centuries, and their daily management go well beyond the permanent exhibition walkthrough.

\section{Keywords}

museum, museography, Monnaie de Paris, collections, money, medal, multimedia 
$\mathrm{H}$ éritier d'une histoire muséale remontant à 1833 , le musée du 11 Conti - Monnaie de Paris s'affirme aujourd'hui comme la référence française la plus récente en termes de muséographie monétaire et médaillistique ${ }^{1}$. Un musée aux accents régaliens légitimes, qui est aussi un musée d'entreprise. Fort de cela le «11 Conti - Monnaie de Paris " aurait pu être un musée de niche. Le nouveau parcours permanent tend à démontrer le contraire, et sa réception par le public - après bientôt un an de fonctionnement - semble le prouver.

\section{D’un musée à l'autre}

La création de l'actuel musée du 11 Conti - Monnaie de Paris s'intègre dans une vaste révision du site historique de la Monnaie de Paris initiée en 2009 par une nouvelle équipe de direction et menée par le cabinet d'architecte Philippe Prost (AAPP). Au nom évocateur de "MétaLmorphoses " ce chantier, qui impacta la quasi-intégralité du vénérable Hôtel de la Monnaie visait - outre un regain de fonctionnalité - à effacer les traces successives et souvent peu heureuses laissées par des passés récents, et à redonner à l'architecture de Jacques Denis Antoine (1733-1801), créateur du lieu entre 1770 et 1775, une lisibilité parfois occultée, souvent saccagée ${ }^{2}$.

Au démarrage de MétaLmorphoses, un musée (deuxième du nom) est accessible au public depuis près de 20 ans dans les halls du "Grand Monnayage » et du «Laminage ». Initialement destiné à accueillir la frappe des monnaies et placé très symboliquement au centre du complexe néoclassique, le " Grand Monnayage » conserva cet usage jusqu'au début des années 1970, date à laquelle les frappes de monnaies de courantes furent délocalisées dans une seconde usine "Monnaie de Paris " : l'Établissement monétaire de Pessac (Gironde). Longtemps utilisée comme salle d'exposition, l'imposante salle accueillit, en 1988, le deuxième musée de

1. Ce qui ne signifie en aucun cas plus audacieuse. Le deuxième musée de la Monnaie de Paris ouvert en 1988 à l'initiative du conservateur de l'époque Jean Belaubre et de Bruno Collin, conçu par les architectes associés Laurent Guynamard, Yves Kneuzé et Bruno Donzet était absolument novateur en termes de muséographie mais aussi de discours assumé. Fermé en 2012 et remplacé par l'actuel musée du 11 Conti, ce musée fait l'objet d'une étude "Economics at the museum: a material history of economics on display $\left(19^{\text {th }}-21^{\text {st }}\right.$ centuries)" menée conjointement par l'université Paris 1 et l'université de Lausanne.

2. À l'instar de la chapelle dont ne reste plus aujourd'hui que le volume et de rares vestiges de décors (dont des peintures en grisaille du peintre Jean-Jacques Forty). 
la Monnaie (Fig. XII, cahier couleur). Après vingt ans de bons et loyaux services, son sort était scellé par la direction nouvellement installée. Les collections furent remisées, les installations supprimées et les lieux livrés aux entreprises avec comme objectif d'en refaire une zone d'ateliers (frappe de médailles, voire d'euros commémoratifs), visible du public depuis un nouveau "Parcours permanent» (Fig. XIII, cahier couleur).

D’emblée, la nécessité de créer un nouveau musée fut mise en avant. Un musée qui, toutefois, ne devait surtout pas en porter la dénomination, jugée passéiste par l'équipe réformatrice ! Parcours muséal, expérientiel, cité du métal... Autant d'appellations en usage tout au long du chantier ${ }^{3}$. Certes, la volonté d'ouvrir davantage le site au public, d'y drainer plus de visiteurs (potentiels clients) et d'en faire une zone de chalandise allait en ce sens. Mais le changement de statut dont la Monnaie de Paris est l'objet à peu près au même moment - passant du statut de Direction du ministère des Finances à celui d'ÉPIC - en faisait concrètement une mission obligatoire $^{4}$. Divisé en trois phases, le chantier MétaLmorphoses consacra la phase 2 au musée dont le chantier démarra en 2012 pour s'achever officiellement le 25 septembre 2017, date d'inauguration du «11 Conti » par Édouard Philippe, Premier ministre et Bruno Le Maire, ministre de l'Économie et des Finances (tutelle directe de l'ÉPIC « Monnaie de Paris»).

Comme pour tout projet de cette envergure, la gestation puis l'accouchement du nouveau musée ne furent pas sans embûche. En vertu d'une politique de rentabilité que rendait nécessaire le nouveau statut, comment ne pas faire de ce musée un show-room ou un lieu éthéré d'autosatisfaction entrepreneuriale ? Le service de la conservation tenante d'une certaine orthodoxie éthique et scientifique (c'est aussi là une de ses missions) y

3. Une nouvelle direction, mise en place en février 2017, à quelques mois de l'ouverture revint sur cette décision et entérina l'appellation musée.

4. En 2007 la Direction des Monnaies et médailles (Monnaie de Paris étant le nom de marque) devint Établissement public industriel et commercial (ÉPIC). L'article 36 de la loi n ${ }^{\circ} 2006-1666$ du 21 décembre 2006 de finances pour 2007 définit les missions patrimoniales de la Monnaie de Paris $(\mathrm{MdP})$ en ses $5^{\circ}$ et $6^{\circ}$ alinéas : " conserver, protéger, restaurer et présenter au public ses collections historiques et mettre en valeur le patrimoine immobilier historique dont il a la gestion ; (..) préserver, développer et transmettre son savoir-faire artistique et technique ». Le contrat pluriannuel d'entreprise 2012-2017 explicite ce point. Parmi les orientations il est stipulé que « la Monnaie de Paris devra poursuivre les objectifs de conservation, protection, restauration et présentation au public des collections historiques, de mise en valeur du patrimoine immobilier historique dont l'établissement a la gestion et de présentation des savoir-faire artistiques et techniques de la Monnaie de Paris ». Une mission patrimoniale que vient renforcer l'appellation « Musée de France ». 
veilla. Plusieurs acteurs de ce chantier, en interne comme en externe (mâ̂trise d'œuvre, maîtrise d'ouvrage, conservation) s'employèrent à œuvrer pour un juste compromis. Si l'on ajoute la nécessaire accessibilité pour tous (personnes à mobilité réduite, mal et non-voyants, etc.), mais aussi les contraintes des espaces dévolus - situés en étage et donc peu propices à l'installation de collections lourdes (mécaniques notamment) - on comprend que le chantier ait été compliqué à négocier et long à mener.

Le message de la direction de l'époque ne souffrait aucune ambiguïté : ne pas refaire un musée au scénario identique au précédent (l'histoire monétaire et économique française, de la Gaule à nos jours). Mais bien plutôt opter pour des entrées thématiques s'enchaînant les unes après les autres en vertu des contraintes d'espaces, de circulations et d'évacuation. Au final, un plan fut proposé par l'équipe de conservation. Âprement discuté, il fut accepté, dans l'ensemble respecté, parfois adapté, souvent complété par une offre multimédia qui permette d'augmenter l'offre informative du parcours tout en lui ajoutant une dimension ludique ; dimension qui manquait assurément dans le musée précédent. Autre époque, autres mœurs...

\section{Le 11 Conti, musée polymatique}

Ce sont au final $1190 \mathrm{~m}^{2}$ de surface utile qui ont été dévolus à la présentation des collections permanentes. Le tout est réparti en huit sections (ou thématiques) :

- Section 1 : Matières premières et Laboratoire,

- Section 2 : La gravure des outillages de frappe,

- Section 3 : La frappe monétaire, du marteau à la presse,

- Section 4 : Les frappes monétaires contemporaines et l'établissement monétaire de Pessac,

- Section 5 : Fonctions et usages de la Monnaie,

- Section 6 : L'art de collectionner,

- Section 7 : Les trésors,

- Section 8 : Productions artisanales et d'exception.

Sur deux niveaux, 1900 objets s'exposent en vertu d'une muséographie qui, quoique moins audacieuse que le précédent musée, y gagne en clarté et en élégance. Souhait de la conservation : mettre le plus possible les objets en scène afin de les rendre d'eux-mêmes plus évocateurs. Textes de salles, 
cartels de vitrines et cartels d'objets, traduits en trois langues viennent compléter le dispositif. Si l'on y ajoute plusieurs vues sur certains ateliers (fonderie, ciselure, patine, frappe), on comprend que le nouveau musée offre plus d'un champ d'entrée, tant pour le visiteur individuel seul, que pour le visiteur en groupe accompagné d'un médiateur.

Section par laquelle on entame le parcours, la salle des matières premières surprend le visiteur de prime abord (Fig. XIV, cahier couleur) ! Sa justification tient à l'histoire scientifique du lieu qui dès son ouverture en 1775 fut pourvu d'un " laboratoire des essais " destiné à s'assurer de la teneur en métaux précieux des alliages. Performant et réputé, le laboratoire devint rapidement, et tout au long des $\mathrm{XIX}^{\mathrm{e}}$ et $\mathrm{XX}^{\mathrm{e}}$ siècles, le théâtre de recherches fondamentales menées par des générations d'essayeurs. Si l'on y ajoute la création en 1778 d'une chaire de minéralogie docimastique ${ }^{5}$ - embryon de la future École royale des mines (1783) elle-même pourvue dès son origine quai Conti d'un cabinet de minéralogie - on comprend combien une telle salle se justifiait. Une manière aussi de renouer avec ce passé scientifique prestigieux que marqueront des noms comme Baltasar Georges Sage (1740-1824), Louis-Joseph Gay-Lussac (1778-1850) ou Alfred Riche (1829-1908).

Axées sur la maîtrise des éléments chimiques du monde métallique, les vitrines reprennent individuellement les métaux clefs du monde des monnaies et médailles : zinc, nickel, étain, cuivre, fer, argent, or et platine, auxquels s'ajoutent les alliages et notamment le bronze. Pour chacun d'entre eux, au moyen de minerais, de monnaies, d'épreuves, d'essais ou encore de médailles, sont expliqués leur gîtologie, l'histoire de leur exploitation, leur relation avec l'univers monétaire, enfin leur lien plus spécifique avec les productions de la Monnaie de Paris. Parmi les nombreux objets phares de cette section les kilogramme et mètre étalon en platine, un lingot d'argent aux poinçons d'Hernan Cortez, une couronne d'or de Philippe de Valois, ou encore la très belle médaille du Val-de-Grâce par Jean Warin.

La section se poursuit par un historique du Laboratoire des essais avec les dates clefs, les grandes figures, mais aussi une mise en scène vidéo des procédés d'analyses, de la coupellation du XviII ${ }^{\mathrm{e}}$ siècle jusqu'à la diffraction $\mathrm{X}$ des années 2000. Pour clore la section : un tableau périodique des

5. Discipline visant à optimiser l'exploitation des minerais, d'or et d'argent notamment. 
éléments interactif axé sur les contraintes physico-chimiques des monnaies et des médailles.

La section 2 met aux prises le visiteur avec le domaine de la gravure, et les notions d'envers et d'endroits, sans la maîtrise desquelles n'existeraient ni monnaies ni médailles. Durant des siècles, la seule méthode de gravure des fers permettant la frappe était la taille directe : attaque à la main, à l'échelle, en creux et à l'envers du motif qui, au moyen de la frappe, se reportera en relief et à l'endroit sur la monnaie ou la médaille. Les outillages (poinçons et matrices, aussi dénommées coins) gravés de l'Ancienne Monnaie de Médailles constituent le clou cette section, tant par leur qualité, que par leur élégante présentation. L'explication de cette méthode se fait au moyen d'un bureau (bien réel) de graveur sur lequel se font de régulières démonstrations. En dehors de celles-ci, une projection immersive tourne en boucle, se substituant habilement aux mains vivantes de l'artisan. Mais la taille directe n'est pas la seule méthode. Au-delà des modelages ou autre gravure laser (expliqués au moyen de vidéos) une méthode retient particulièrement l'attention : la réduction. Trônant fièrement au milieu de la salle, le tour à réduire se distingue par sa mécanique quasi mathématique. Compromis entre le tour axial et le pantographe, le tour à réduire (inventé à la fin du XIX ${ }^{e}$ siècle) lit un modèle (ou gabarit) et en reporte les dessins et creux sur un bloc d'acier (la future matrice) au moyen d'une gravure faite simultanément. Le caractère et l'histoire de ce pantographe en 3D, en font assurément la machine la plus spécifique à l'univers des monnaies et médailles.

La section 3 (Fig. XV, cahier couleur) retrace l'histoire de la frappe monétaire qui, par son ancienneté et sa nature régalienne, constitue un pôle d'intérêt majeur du parcours. Une histoire en trois parties qui s'ouvre par la frappe au marteau. Pratiquée de l'Antiquité jusqu'au XvII ${ }^{\mathrm{e}}$ siècle, la méthode est évoquée au moyen de rarissimes outillages, de monnaies, mais aussi de deux vitraux du $\mathrm{XvI}^{\mathrm{e}}$ siècle représentant l'intérieur d'atelier monétaire. C'est au Xvi ${ }^{\mathrm{e}}$ siècle que sont pointées du doigt les insuffisances de cette technique : rognage, imitation et finalement fraude massive menacent la souveraineté et l'économie des États. La frappe au balancier, commencée en France sous le règne d'Henri II, tente de subvenir à ces nouveaux besoins. En raison des contraintes liées aux résistances des sols, les plus belles pièces mécaniques de la collection ne purent être intégrées à cette section. À commencer par les deux balanciers historiques de De Launay 
destinés - dès leur origine en 1698 et 1699 - à la frappe des jetons et à celle des médailles de l'Histoire métallique de Louis XIV. Contraint de se passer de deux monuments majeurs du patrimoine numismatique national, le nouveau musée peut néanmoins se prévaloir de présenter des machines plus modestes en taille mais d'un intérêt non moindre. Ainsi le balancier de Brézin, conçu pour l'Hôtel de la Monnaie sur des dessins d'Antoine. Ou encore un exemplaire d'un des balanciers « d'Austerlitz " conçus en 1807 par Philippe Gengembre. Témoignant de cette technique dont la mâ̂trise alla croissant du XVI ${ }^{\mathrm{e}}$ au XIX ${ }^{\mathrm{e}}$ siècle, les pièces d'or de 10 louis - dues en 1640 au talentueux graveur Jean Warin - attirent tous les regards par leur taille et la perfection de leur fabrication. Pour clore la section, l'ère de la Presse qui, à partir des années 1810-1840, et jusqu’à nos jours, fait de la fabrication monétaire une opération industrielle et à la rentabilité accrue. Marquage de la tranche, bimétallisme viennent compléter sous forme de focus ce panorama de vingt siècles.

À noter que pour cette section l'apport multimédia est impressionnant : chronologie des techniques (incluant celles non retenues en France, mais explorées en d'autres États), films, hand-zones. Parmi ces dernières, une opération de frappe au marteau permet au visiteur de se frotter à cette technique finalement plus délicate qu'il y parait ${ }^{6}$. Loin d'un simple amusement, ce dispositif permet de connaître sa force de frappe, mais aussi son aptitude à frapper dans l'axe et dans le plan des outillages, pour une monnaie régulière en centrage et en épaisseur (Fig. 1). Frappant de pédagogie ! Cette section 3 s'achève symboliquement en 1972, année de création de l'Établissement monétaire de Pessac (Gironde) qui constitue le sujet de la section suivante (4), seule section à se trouver en rez-de-chaussée. Ce « Nouveau temple de Junon Moneta » qui répondait à la nécessité impérieuse pour la Monnaie de Paris de s'agrandir et de se moderniser, est évoqué par plusieurs vitrines reprenant les trois grandes étapes du process monétaire moderne : la gravure et la démultiplication (longtemps par enfonçage) des coins (matrices monétaires) ; la fabrication en parallèle, par découpe, des flans (rondelles de métal vierge) ; la frappe proprement dite des flans sur les coins donnant naissance aux pièces de monnaies, françaises (opération régalienne) et étrangères (opération sous le régime du gré à gré

6. Conception : Du\&Ma \& Monnaie de Paris. Réalisation : A.V.É.A.M. \& Opixido. 
ou de la concurrence). Un film, enrichi d'une infographie évolutive, complète l'évocation du lieu.

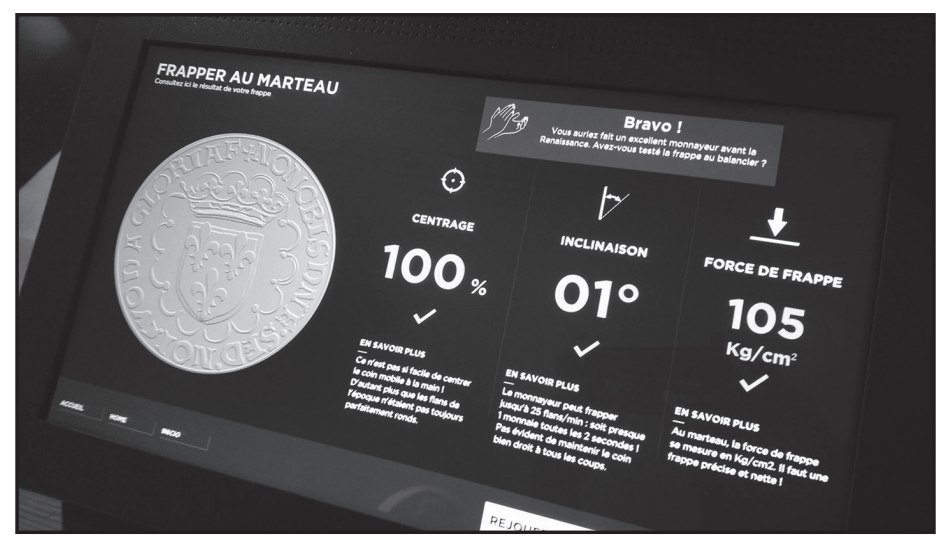

Fig. 1. - Le dispositif interactif de frappe au marteau, 2018

(c) Du\&Ma \& Monnaie de Paris / A.V.É.A.M. \& Opixido

Adjacente, la rotonde du Grand Monnayage sur lequel veille la statue de la Fortune par Mouchy renvoie aux temps où la monnaie y était frappée : forges, flaons, espèces... la "signalétique " gravée en lettres d'or dans des cartouches de marbre gris rappelle la fonction initiale du lieu. Un film riche de nombreux documents d'archives collectés pour l'occasion évoque en images l'histoire du lieu. De là, et par une grande baie vitrée, le visiteur appréhende de plain-pied et d'un seul coup d'œil le Grand Monnayage proprement dit où se dressent aujourd'hui d'imposantes presses pour la frappe de médailles et d'euros commémoratifs.

La remontée à l'étage permet d'accéder à la cinquième section, où la monnaie est abordée sous l'angle de ses formes, fonctions et usages. Où l'on découvre que la monnaie n'a pas toujours été ronde et en métal. Afrique et Asie témoignent ainsi d'autres formes et matières. Nécessité faisant loi, les monnaies en temps de guerres changent elles aussi d'aspect et leurs matériaux se diversifient. En périodes plus favorables, les monnaies tendent vers l'union (Union Latine, Zollverein, Euro). Quand l'une d'entre elles - forte d'un crédit de confiance plus important - ne tente pas de supplanter les autres (Gros sous, florin, Ducat, Thaler, dollar...) ! Récompensé par 
un prix en $2017^{7}$, le numiscope (multimédia basé sur la technologie 3D) permet de zoomer sur une sélection de dix monnaies emblématiques et d'en déchiffrer les iconographies et légendes.

Les deux dernières sections font en quelque sorte sortir la monnaie du circuit d'échange, pour devenir objet de collection ou de thésaurisation.

À partir d'exemples notoires (Louis XIV, Henri Abel, ou collectionneurs contemporains), l'art de la collection nous est révélé. Voir ainsi dans le tableau de François Lemaire, Jean Warin initier le jeune Louis XIV à la "science des médailles " en lui montrant une monnaie d'Alexandre Le Grand, n'a clairement rien d'anodin! Ou quand la monnaie devient objet de pédagogie.

L'évocation de la monnaie comme objet de thésaurisation, passe quant à elle par une "chambre forte " à la mise en scène résolument assumée. Quatre typologies de trésors sont ainsi présentées : trésor de périodes troublées (trouvaille romaine des Authieux), trésor marin (lingots et monnaies d'argent de l'épave du Slot ter Hooge), héritage dissimulé (trésor de louis d'or de la rue Mouffetard à Paris), prise de guerre (trésor de Hué). Bien plus que la quantité, et au-delà de nos imaginaires parfois réducteurs, la section montre la diversité que peuvent revêtir les trésors. À noter la présentation audacieuse du pondéreux trésor de Hué, constitué de lingots et de médaillons d'or, dont l'exposition au grand public est une première.

À l'issue de ces sixième et septième sections, le visiteur achève son parcours avec la présentation des productions contemporaines (autres que monétaires) de la Monnaie de Paris : médailles, bijoux, fontes d'art... Membre du Comité Colbert, la Monnaie perpétue de nobles savoir-faire en matière de frappe, de fonte, de patine ou encore d'émaillage. Au-delà des objets exposés, la chaîne opératoire menant de la matière brute à l'objet fini constitue un axe fort de la présentation : les différentes étapes de frappes et de recuits des médailles y sont par exemple clairement palpables par le visiteur. Quant aux hommes et aux femmes, artisans à l'origine de chacun de ces objets, ils occupent une place de choix dans cette ultime section grâce à un imposant apport vidéo.

7. Primé dans la catégorie Innovative Application au Festival of Audiovisual International Multimedia Patrimony 2018, organisé par l'AVICOM, l'ICOM International Committee for Audiovisual, New Technologies and Social Media. 


\section{Pédagogique par l'objet}

Conçu dans une démarche d'exhaustivité, et tentant de ne jamais sacrifier la qualité intellectuelle du propos, le musée du 11 Conti - Monnaie de Paris permet à chacun d'appréhender l'univers des monnaies et médailles par l'angle qui lui plaira : scientifique ou technique, artistique ou économique. Ajoutons les expositions-dossiers qui animeront le propos au cœur même du musée, mettant en avant les collections non exposées, les travaux scientifiques des personnels de la Conservation ou encore les résultats des opérations légales de récolement, sources souvent de belles redécouvertes.

Bien sûr, et comme pour toute chose, le lieu est perfectible. D'aucuns reprocheront que le 11 Conti ne présente aucunement, et de manière continue, l'histoire monétaire de notre pays. Cette piste ayant été rejetée en son temps, une frise s'y substitue, sans satisfaire personne. À l'heure de la démocratie participative, le public n’hésite pas à pointer du doigt ce manque. Mais le musée se veut évolutif. Son éditorial pourra s'adapter aux volontés internes de l'institution et aux besoins exprimés des publics. Et pour cela le musée peut compter sur une collection exceptionnelle conservée au cœur d'un cadre non moins exceptionnel. Un lieu non pas tant numismatique que pédagogique... La pédagogie par l'objet que ni Jean Warin, ni B.G. Sage, figures tutélaires des lieux, ne démentiraient ${ }^{8}$.

8. L'auteur remercie Sylvie Juvénal, chargée de Fonds patrimoniaux de la Monnaie de Paris pour les corrections et compléments qu'elle a bien voulu apporter à ce texte, et Pernille Soldat pour ses traductions. 